\title{
Effect of Experimentally Observed Hot Atom Adsorption Mechanism on the Phase Diagram of Monomer-Dimer Catalytic Reaction on Pt(111): A Monte-Carlo Simulation Study
}

\author{
M. Khalid ${ }^{1}$, Q. N. Malik ${ }^{1}$, A. U. Qaisrani ${ }^{1}$, and M. K. Khan ${ }^{2}$ \\ ${ }^{1}$ Department of Physics Gomal University, Dera Ismail Khan, Pakistan and \\ ${ }^{2}$ Department of Physics, Quaid-i-Azam University, Islamababd, Pakistan
}

Received on 18 March, 2005

\begin{abstract}
The effect of experimentally observed hot atom mechanism on the phase diagram using Pt (111) surface of the monomer-dimer reaction has been studied. While using this mechanism, whenever $\mathrm{O}_{2}$ molecule hits the randomly vacant selected site, it breaks up into atomic form and then executes ballistic flight. The path of the two oxygen atoms is taken exactly opposite to each other i.e. anti-parallel and range of the atoms is taken equal i.e. they may fly upto 1 and 1.73 atomic spacing from the site of impact. Two cases have been studied on the basis of the range of hot atoms. The steady reactive window is observed and the continuous transition disappears. As soon as the $\mathrm{CO}$ partial pressure departs from zero the production of $\mathrm{CO}_{2}$ is observed, which clearly verifies the experimental observation.
\end{abstract}

Keywords: Catalytic surface reactions; Monte Carlo simulation; Adsorption mechanism; Heterogeneous catalysis

\section{INTRODUCTION}

The reaction of carbon monoxide on transition metal surfaces was one of the first reactions studied by surface science methods [1]. Since that time oxidation of carbon monoxide has served as the most important and best understood model for surface reactions. Moreover, the adsorption of $\mathrm{CO}$ on platinum surfaces has been extensively studied due to its chemical and industrial importance in various catalytic processes. Lattice models of catalytic processes have been used successfully to predict a wide range of experimental observations in catalysis and have been extremely helpful in gaining a better understanding of the kinetics of catalytic processes [2]. Many experimental and theoretical works for the $\mathrm{CO}$ catalytic oxidation system are given in the literature [3], but Monte- Carlo Simulation has been widely used to investigate the details of this reaction system. Computer simulations have been used extensively to explore adsorption, desorption, and phase transitions reaction kinetics and other aspects of surface chemistry and physics. In particular, interest is growing to develop a better understanding of heterogeneous catalysis [4-6]. In this regard, the pioneering work has been done by Ziff, Gulari and Barshad (ZGB) [7]. They introduced a monomer-dimer (MD) model, which has been used to study a reaction system of the type $2 \mathrm{~A}+\mathrm{B}_{2} \rightarrow 2 \mathrm{AB}$. In this reaction $\mathrm{A}$ is $\mathrm{CO}, \mathrm{B}_{2}$ is $\mathrm{O}_{2}$ and $\mathrm{AB}$ is $\mathrm{CO}_{2}$. . This model is generally known as the ZGB model. Details of this seminal work can be found in the literature elsewhere $[7,8]$. The reaction mechanism exhibits two phase transitions $\mathrm{y}_{1}$ and $\mathrm{y}_{2}$. If the feed concentration of $\mathrm{CO}$ $\left(\mathrm{y}_{C O}\right)$ is less than $\mathrm{y}_{1}$, the surface is completely covered with oxygen and if the feed concentration of $\mathrm{CO}$ is greater than $\mathrm{y}_{2}$, the lattice is totally filled with CO. Only in the range $\mathrm{y}_{1}<\mathrm{y}_{C O}$ $<\mathrm{y}_{2}$ does the system exhibit a steady reactive state (SRS). The transition at $\mathrm{y}_{1}$ is continuous while at $\mathrm{y}_{2}$ it is discontinuous. Here $\mathrm{y}_{1}$ is the critical concentration of $\mathrm{CO}$ at which an SRS starts, while $\mathrm{y}_{2}$ is the critical concentration of $\mathrm{CO}$ where an SRS stops. Experiments show that the production of $\mathrm{CO}_{2}$ starts as soon as $y_{C O}$ departs from zero [9-11]. Inspired by the work of Ziff et al [7], different authors have studied variants of the ZGB model [12-27] .

Recent experiments by scanning tunneling microscopy (STM) on the dissociative adsorption of oxygen molecules on an $\mathrm{Al}$ (111) surface [28] pointed to a "hot atom" mechanism according to which the two oxygen atoms are propelled apart upon dissociation by distances exceeding $80 \AA$ in a ballistic type of motion before equilibrating with the substrate. The hot oxygen atoms react with adsorbed $\mathrm{CO}$ molecule or can stimulate desorption of other co- adsorbed particles [28-30]. Wintterlin et al [29] showed existence of a hot atom mechanism for the dissociation of $\mathrm{O}_{2}$ on $\mathrm{Pt}$ (111) surface through variable temperature STM. Pt (111) surface can be approximated to a two dimensional hexagonal lattice where first nearest neighboring (nn) sites have a distance of one atomic spacing.

The objective of this work is to study the influence of experimentally observed hot atom mechanism on the phase diagram of $\mathrm{A}-\mathrm{B}_{2}$ reaction system. We have chosen the hexagonal lattice $\mathrm{Pt}$ (111) for this study. The effect of the reaction between a hot oxygen atom and CO-adsorbed (A ) atom on the phase diagram of the system has also been studied.

\section{MODEL AND SIMULATION}

The usual simple LH surface model of the system can be written in the form of the following equations:

$$
\begin{gathered}
\mathrm{A}(\mathrm{g})+\mathrm{S} \rightarrow \mathrm{A}^{S} \\
\mathrm{~B}_{2}(\mathrm{~g})+\mathrm{S} \rightarrow \mathrm{B}^{b}+\mathrm{B}^{b}+\mathrm{S} \\
\mathrm{B}^{b}+\mathrm{S} \rightarrow \mathrm{B}^{S}
\end{gathered}
$$




$$
\begin{aligned}
& \mathrm{A}^{s}+\mathrm{B}^{s} \rightarrow \mathrm{AB}(\mathrm{g})+2 \mathrm{~S} \\
& \mathrm{~B}^{b}+\mathrm{A}^{s} \rightarrow \mathrm{AB}(\mathrm{g})+\mathrm{S}
\end{aligned}
$$

Where $(\mathrm{g})$ indicates the species in the gas phase and $\mathrm{S}$ represents a vacant surface site. $\mathrm{X}^{b}$ and $\mathrm{X}^{S}$ represent the atom executing ballistic motion and the adsorbed species, respectively.

The relative impingement rates of $\mathrm{A}$ and $\mathrm{B}_{2}$ species, which are proportional to their partial pressures are normalized $\left(\mathrm{Y}_{A}\right.$ $+\mathrm{Y}_{B}=1$ ), so the model has a single parameter, i.e. $\mathrm{Y}_{A}$.

We consider an infinite reservoir filled with $\mathrm{A}_{\text {and }} \mathrm{B}_{2}$ with partial pressures $\mathrm{Y}_{A}$ and $1-\mathrm{Y}_{A}$, respectively. This reservoir is in contact with a surface which is simulated by means of a hexagonal lattice of linear dimension $\mathrm{L}=128$. It is observed that an increase of the lattice size can change the critical pressures slightly but the overall qualitative nature of the phase diagram is not affected [31]. Periodic boundary conditions are utilized in order to avoid boundary effects. The simulation starts with a clean surface. The equilibrium coverages are measured as a function of $\mathrm{Y}_{A}$. In order to locate the critical points ten independent runs each up to 50,000 Monte Carlo (MC) cycles are carried out. If all the ten runs proceed up to 50,000 MC cycles without the lattice getting poisoned, the particular point is considered to be within SRS. The poisoning of even a single run is a sufficient criterion for considering the point to belong to the poisoned state. If the run does not end up in a poisoned state, then in order to get the coverages corresponding to the SRS, the initial 10,000 MC cycles are disregarded and averages are taken over the subsequent 40,000 MC cycles. The values of coverages (production rate) are obtained after every $10 \mathrm{MC}$ cycles, so that the final coverage (production rate) is an average taken over 4000 configurations.

The simulation proceeds as follows: There are two possibilities for the randomly selected site (i) site might be occupied or (ii) vacant. The trial ends if the selected site is occupied. If the site is vacant and A molecule happens to be selected, then adsorption is carried out via reaction step (1). After adsorption, A molecule goes for reaction step (4). If $\mathrm{A}^{S}$ finds B in its nearest neighbors, then $\mathrm{AB}(\mathrm{g})$ is produced with the creation of two vacancies otherwise trial ends.

If the selected molecule is $\mathrm{B}_{2}$ and selected site is vacant, then the $\mathrm{B}_{2}$ molecule dissociates into two hot atoms via reaction step (2). These two hot atoms execute a ballistic flight and fly in opposite direction (anti-parallel) from the site of impact and their range is taken to be equal, i.e. both atoms may fly up to the first nearest ( 1 atomic spacing from the site of impact, case a) or up to second nearest neighbors (1.73 atomic spacing from the site of impact, case b). Two hot atoms may take one of the following courses: (i) if the selected site (one or both) is occupied by B, then trial will end; (ii) if both the sites are vacant, then both the $\mathrm{B}$ atoms will be adsorbed via reaction step (2). After adsorption both adsorbed oxygen atoms go for reaction step (4). If $\mathrm{B}$ atom (one or both) finds $\mathrm{A}^{S}$, in its first nearest neighbors, then $\mathrm{AB}(\mathrm{g})$ is produced with the creation of two vacancies, otherwise trial ends. (iii) If one site is vacant and $\mathrm{A}$ occupies the other then one $\mathrm{B}^{b}$ atom is adsorbed via reaction step (3) and then goes for reaction step (4). The other
$\mathrm{B}^{b}$ will directly react with $\mathrm{A}^{S}$ via step (5). (iv) If A occupies both randomly selected sites, then both $\mathrm{B}^{b}$ atoms go through reaction step (5). The output of the reaction step (5) is $\mathrm{AB}(\mathrm{g})$ with the creation of one vacancy.

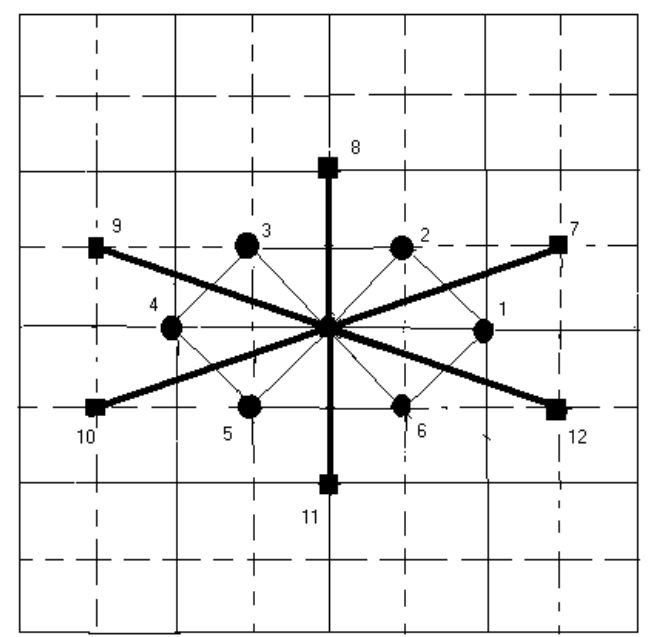

FIG. 1: Surface and subsurface layers of Hexagonal lattice. The sites marked by 1-6 are first $\mathrm{nn}$ at a distance of 1 atomic spacing; and the sites marked by 7-12 are second $\mathrm{nn}$ at a distance of 1.73 atomic spacing.

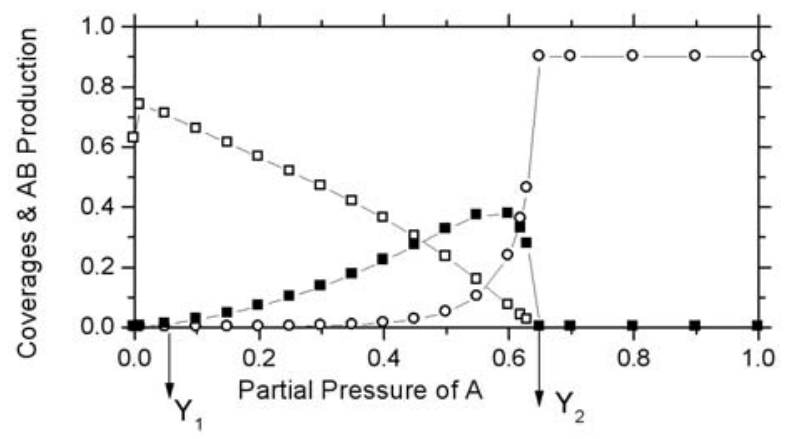

FIG. 2: Coverage's of B (open square), A (open circle) and the production rate of $\mathrm{AB}$ (solid square) versus $\mathrm{A}$ partial pressure for case (a).

\section{RESULTS AND DISCUSSION}

Figures 2 and 3 show the coverages of the species and production of $A B$ plotted as a function of $Y_{A}$ for case (a) and case (b) respectively. In ZGB model a pair of vacancy in the first nearest neighbourhood is required for impingement and consequent dissociation of the $\mathrm{B}_{2}$ molecule. The trial ends if a pair of vacancy is not available. On the other hand, in the present model only a single site is required for impingement and the consequent dissociation of $\mathrm{B}_{2}$ molecule. This 


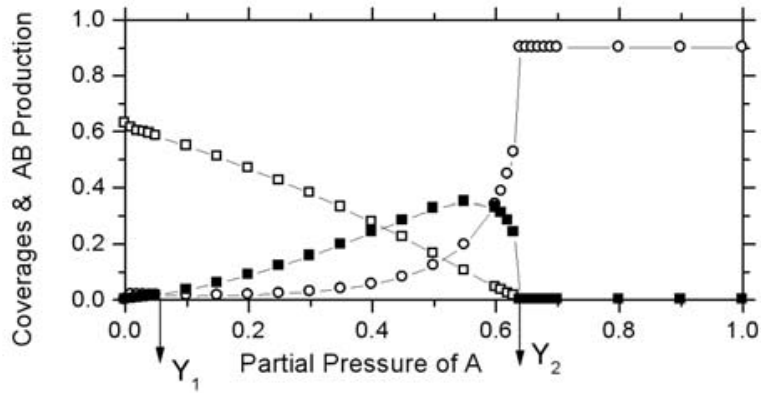

FIG. 3: Coverages of B (open square), A (open circle) and the production rate of $\mathrm{AB}$ (solid square) versus $\mathrm{A}$ partial pressure for case $\mathrm{b}$.

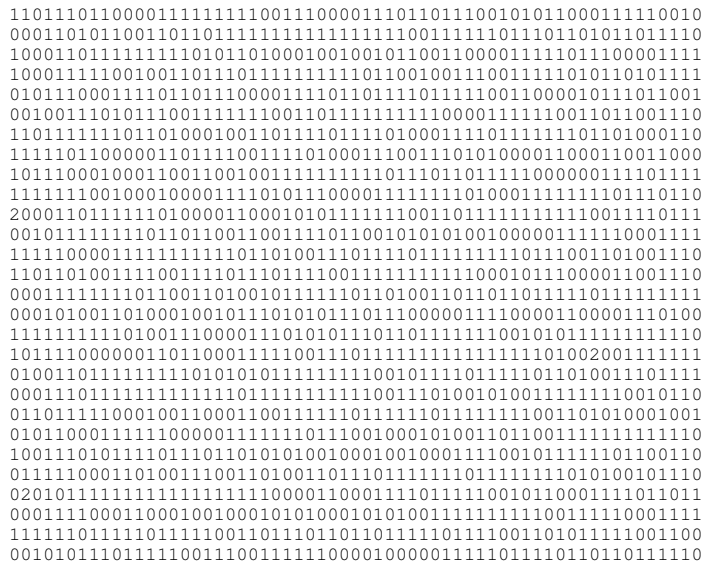

FIG. 4: Snap shot on $\mathrm{y}_{c o}=0.14$ ( 1 for $\mathrm{B}, 2$ for $\mathrm{A}$ and 0 for vacant site).

change cannot affect the situation when the partial pressure of the species $\mathrm{B}_{2}$ is high ( $\mathrm{A}$ is low). This is because in this region no $\mathrm{A}$ is available to adsorb on the surface (every $\mathrm{CO}$ impinging on the surface is reacted and burnt); only $\mathrm{B}_{2}$ molecules are available for adsorption on the surface. That is why the value of $\mathrm{y}_{1}$ (where $\mathrm{y}_{1}\left(\mathrm{y}_{2}\right)$ is the partial pressure or feed concentration of the monomer at which the production starts (terminates)) and the qualitative nature of the phase diagram observed in our model is similar to that of the ZGB model. However, the requirement of a single vacancy for accommodation of molecule of $\mathrm{B}_{2}$ in contrast to the ZGB model plays an important role in the region where $A$ starts adsorbing on the surface. In the ZGB model, when the supply of oxygen is limited due to unavailability of a pair of vacancies then A starts occupying the single vacancies whereas in the present model only a single vacancy is required to accommodate both species ( $B_{2}$ and $A$ ) on the surface. So, naturally in contrast to ZGB model the reaction will be sustained to a higher value of feed concentration, which results in the shift of $y_{2}$ from 0.525 (in $\mathrm{ZGB}$ ) to 0.65 . It is fairly known that at high partial pressure, $\mathrm{B}_{2}$ forms large islands (Fig. 4). In this situation, if you pick a single vacancy as a site of impact, then there is a small chance that the other vacancy at a distance mentioned above can be obtained. Under these conditions, every trial of $\mathrm{B}_{2}$ impingement will end unsuccessfully. However, in the same situation every trial of A impingement will end in the burning of oxygen. This means that indirectly, adsorption (supply) rate of $\mathrm{B}_{2}$ is decreased whereas that of $\mathrm{A}$ is increased. This fact will shift the value of $y_{1}$ towards lower feed concentration. That is why the value of $\mathrm{y}_{1}$ in all the two cases has been shifted from 0.39 to 0.05 . However, the qualitative nature of the phase diagrams are similar for case (a) and (b). It is clear in Figs. 1 and 2 that for low concentration of $\mathrm{A}$, the elimination of the second-order phase transition takes place and the production of $\mathrm{AB}$ starts the moment the feed concentration departs from zero. In this situation the phase diagram closely resembles the experimental one qualitatively.

The experimental observation of Wintterlin et al [29] that the chance of dissociative adsorption of $\mathrm{B}_{2}$ molecule on a pair of nearest neighbouring sites (like ZGB model) is very small seems to be almost true in our simulation as it does give a phase diagram (Figure 2,3) comparable to the real one [32]. However, the phase diagrams shown in Figure 2 and 3 closely resemble to that of experimental one and are consistent with the observation of Wintterlin et al [29] that the two hot oxygen atoms appear in pairs.

\section{CONCLUSION}

In conclusion, we have studied catalytic oxidation of A on a Pt (111) on the basis of a ballistic type mechanism. The effect of this adsorption mechanism on the phase diagram of ZGB model have been studied. This model depicts a situation, which is observed in experiments i.e. the production of $A B$ starts the moment A partial pressure departs from zero. The phase diagram becomes qualitatively similar to that observed experimentally for the reaction system.
[1] G. Ertl and P. Rau, Surf. Sci. 15, 443 (1969).

[2] R. Imbihl and G. Ertl, Chem. Rev. 95, 697 (1995).

[3] T. Engel and G. Ertl, Adv. Catal. 2, 28 (1997).

[4] M. Silverberg et al, J. Chem. Phys. 87, 3178 (1987).

[5] B. Chopard, Mdroz, J. Phys. A21, 205 (1988).

[6] X. P. Jiang and H. Metiu, J. Chem. Phys. 88, 1891 (1988).

[7] R. M. Ziff, E. Gulari, and Y. Barshad, Phys. Rev. Lett. 56, 2553 (1986).
[8] K. M. Khan, A. Basit, and K. Yaldram, J. Phys. A 33, L215 (2000).

[9] H. H. Rotermund, J. Lauterbach, and G. Haas, Appl. Phys. A57, 507 (1993).

[10] S. Ladas, R. Imbihl, and G. Ertl, Surf. Sci. 219, 88 (1989).

[11] P. Meakin and D. J. Scalapino, J. Chem. Phys. 87, 731 (1987).

[12] V. Schmatloch, I. Jirka, S. Heinze, and N. Kruse, Surf. Sci. 331, 23 (1995). 
[13] I. Jensen and H. C. Fogedby, Phys. Rev. A 42, 1969 (1990).

[14] J. W. Evans, J. Chem. Phys. 98, 2463 (1993).

[15] F. Bagnoli, B. Sente, M. Dumont, and R. Dagonnier, J. Chem. Phys. 94, 777 (1991).

[16] K. M. Khan and N. Ahmad, Physica A 280, 391 (2000).

[17] K. M. Khan and K. Yaldram, Surf. Sci. 445, 186 (2000).

[18] K. M. Khan, Surf. Sci. 470, 155 (2000).

[19] N. Ahmad and K. M. Khan, Chem. Phys. 263, 339 (2001).

[20] P. Meakin, J. Chem. Phys. 93, 2903 (1990).

[21] K. M. Khan and W. Ahmed, J. Physica A 35, 2713 (2002).

[22] R. Imbihl and G. Ertl, Chem. Rev. 95, 697 (1995).

[23] A. U. Qaisrani, M. Khalid et al, Chin. Phys. Lett. 21, 1838 (2004).

[24] M Khalid, K. M. Khan, A. U. Qaisrani, and Q. N. Malik, Chin. Phys. Lett. 21, 1171 (2004).

[25] M. Khalid and A. U. Qaisrani, Int. J. Mod. Phys. C 15, 1215
(2004).

[26] K. M. Khan and E. V. Albano, Chem. Phys. 276, 129 (2002).

[27] T. Tome and R. Dickman, Phys. Rev. E 47, 948 (1993).

[28] H. Brune, J. Wintterlin, J. Trost, G. Ertl, Wiechers R. J. Behm, Chem. Phys. 99, 2126 (1993).

[29] J. Wintterlin, R. Schuster, and G. Ert, Phys. Rev. Lett. 77, 123 (1996).

[30] A. N. Artsyukhovich and I. Harrison, Surf. Sci. 350, 1199 (1996).

[31] K. M. Khan, K. Yaldram, and N. Ahmad, J. Chem. Phys. 109, 5054 (1998).

[32] E. V. Albano, Computational Methods in Surface and Colloid Science, Ed. M. Borowko, (Marcel Dekker, New York), 387(2000). 